\title{
Characterization of Pancreatic Tumors with Quantitative Perfusion Analysis in Contrast- Enhanced Harmonic Endoscopic Ultrasonography
}

\author{
Shunsuke Omoto ${ }^{a}$ Mamoru Takenaka ${ }^{a}$ Masayuki Kitanod ${ }^{d}$ Takeshi Miyata $^{a}$ \\ Ken Kamata ${ }^{a}$ Kosuke Minaga ${ }^{a}$ Tadaaki Arizumia Kentaro Yamao ${ }^{a}$ \\ Hajime Imai $^{a}$ Hiroki Sakamoto ${ }^{a}$ Yogesh Harwani ${ }^{a}$ Toshiharu Sakurai ${ }^{a}$ \\ Tomohiro Watanabe $^{a}$ Naoshi Nishida ${ }^{a}$ Yoshifumi Takeyamab \\ Yasutaka Chibac Masatoshi Kudo ${ }^{\mathrm{a}}$ \\ Departments of a Gastroenterology and Hepatology and ${ }^{b}$ Surgery, and ${ }^{\mathrm{C}}$ Division of Biostatistics, Clinical Research \\ Center, Kindai University Faculty of Medicine, Osaka-Sayama, and d Second Department of Internal Medicine, \\ Wakayama Medical University School of Medicine, Wakayama, Japan
}

\section{Keywords}

Contrast-enhanced harmonic endoscopic ultrasonography · Quantitative perfusion analysis · Pancreatic carcinoma ·

Time-intensity curve

\begin{abstract}
Objectives: This study evaluated whether quantitative perfusion analysis with contrast-enhanced harmonic $(\mathrm{CH})$ endoscopic ultrasonography (EUS) characterizes pancreatic tumors, and compared the hemodynamic parameters used to diagnose pancreatic carcinoma. Methods: $\mathrm{CH}$-EUS data from pancreatic tumors of 76 patients were retrospectively analyzed. Time-intensity curves (TIC) were generated to depict changes in signal intensity over time, and 6 parameters were assessed: baseline intensity, peak intensity, time to peak, intensity gain, intensity at $60 \mathrm{~s}\left(\mathrm{I}_{60}\right)$, and reduction rate. These parameters were compared between pancreatic carcinomas ( $n=41)$, inflammatory pseudotumors $(n=14)$, pancreatic neuroendocrine tumors $(n=14)$, and other tumors $(n=7)$. All
\end{abstract}

6 TIC parameters and subjective analysis for diagnosing pancreatic carcinoma were compared. Results: Values of peak intensity and $\mathrm{I}_{60}$ were significantly lower and time to peak was significantly longer in the groups with pancreatic carcinomas than in the other 3 tumor groups $(p<0.05)$. Reduction rate was significantly higher in pancreatic carcinomas than in pancreatic neuroendocrine tumors $(p<0.05)$. Areas under the receiver-operating characteristic curves for the diagnosis of pancreatic carcinoma using subjective analysis, baseline intensity, peak intensity, intensity gain, $\mathrm{I}_{60}$, time to peak, and reduction rate, were $0.817,0.664,0.810,0.751,0.845$, 0.777 , and 0.725 , respectively. $I_{60}$ was the most accurate parameter for differentiating pancreatic carcinomas from the other groups, giving values of sensitivity/specificity of 92.7/68.6\% when optimal cutoffs were chosen. Conclusions: In pancreatic carcinomas, TIC patterns were markedly different from the other tumor types, with $\mathrm{I}_{60}$ being the most accurate diagnostic parameter. Quantitative perfusion analysis is useful for differentiating pancreatic carcinomas from other pancreatic tumors.

(c) 2017 S. Karger AG, Basel

\section{KARGER}

(C) 2017 S. Karger AG, Basel

E-Mail karger@karger.com

www.karger.com/ocl
Dr. Mamoru Takenaka

Department of Gastroenterology and Hepatology

Kindai University Faculty of Medicine

377-2 Ohnohigashi, Osaka-Sayama, Osaka 589-8511 (Japan)

E-Mail mamoxyo45@gmail.com 


\section{Introduction}

The number of reports on the utility of contrast-enhanced harmonic $(\mathrm{CH})$ endoscopic ultrasonography (EUS) in the differential diagnosis of pancreatic masses has been increasing [1-5]. CH-EUS depicted the hypoenhancement of pancreatic carcinomas with high sensitivity (89-96\%) and specificity (64-94\%) [1-5]. Nonetheless, the visual evaluation of CH-EUS scans may be influenced by the endosonographers' subjective impressions [6]. In addition, there was no standardized method to analyze the images after the infusion of an ultrasound contrast agent, particularly regarding how to determine the enhancement pattern of the EUS-depicted pancreatic masses.

Recent studies have described quantitative perfusion analysis of pancreatic diseases using a time-intensity curve (TIC), which depicts the changes in signal intensity over time within a region of interest (ROI) after infusion of an ultrasound contrast agent [7-12]. However, the scanning methods and the parameters evaluated varied between these reports. Therefore, the primary aim of this study was to determine if quantitative perfusion analysis with CH-EUS helps to characterize pancreatic tumors. The secondary aim was to find the most accurate hemodynamic parameter of TIC for differentiating pancreatic carcinoma from other pancreatic tumors.

\section{Patients and Methods}

\section{Patients and Study Design}

This retrospective study included patients with a suspect pancreatic mass on the basis of results from computed tomography (CT), magnetic resonance imaging, or transabdominal ultrasonography (US), and who underwent both standard EUS and $\mathrm{CH}$ EUS between February 2011 and February 2012 at the Department of Gastroenterology and Hepatology, Kindai University Faculty of Medicine. Patients were enrolled if the solid component of the mass was $>75 \%$ of the total volume and if, after EUS examinations, they had undergone surgery or EUS-guided fine-needle aspiration (EUS-FNA) leading to a histological or cytological diagnosis, with a follow-up of at least 12 months. Pancreatic cystic tumors with a content of solid tumor $<25 \%$ were excluded. The histological or cytological outcome was considered the gold standard diagnosis for the purposes of this study and was used to divide patients into 4 groups: pancreatic carcinomas, pancreatic neuroendocrine tumors, inflammatory pseudotumors, and other tumors.

\section{Endoscopic Ultrasonography}

Conventional EUS of the pancreas without a contrast agent was performed first, giving special attention to pancreatic masses. When conventional EUS revealed a solid lesion, images of the ideal scanning plane were displayed to portray the whole area of the lesion. Thereafter, the imaging mode was changed to the extended pure harmonic detection mode. This mode synthesizes the filtered second harmonic components with signals obtained from the phase shift, which is used for $\mathrm{CH}$ imaging. The transmitting frequency and mechanical index were $4.7 \mathrm{MHz}$ and 0.3 , respectively. The ultrasound contrast agent Sonazoid (Daiichi-Sankyo, Tokyo, Japan) was used. Immediately before CH-EUS, the contrast agent was reconstituted with $2 \mathrm{~mL}$ of sterile water for injection, and a dose of $15 \mu \mathrm{L} / \mathrm{kg}$ body weight was prepared in a $1-\mathrm{mL}$ syringe. A bolus injection of the contrast agent was administered. With a frame rate of about $10 \mathrm{images} / \mathrm{s}$, all images and hemodynamic data were acquired using a ProSound Alpha 10 US system (Aloka, Tokyo, Japan). All EUS procedures were performed by 2 endosonographers (M. Kitano and H. Sakamoto). One was responsible for endoscopic manipulation and scanning, and the other for operating the US scanner. Both endosonographers are certified by the Japan Gastroenterological Endoscopy Society and have $>10$ years of experience with CH-EUS; each has performed $>1,000 \mathrm{CH}$-EUS procedures. Subjective analysis was performed by these endosonographers for diagnosing pancreatic carcinoma. A hypo-enhancement pattern defined pancreatic carcinoma.

\section{TIC Analysis}

The acquired images were reviewed using the software "Time Intensity Curve" (ProSound Alpha-10 US system; Aloka, Tokyo, Japan). An ROI was placed over the pancreatic mass to cover an area as large as possible, and a TIC was generated to depict the changes in signal intensity over time within the ROI (Fig. 1). From these data, the software calculated the baseline intensity $(\mathrm{dB})$, and, then, after the injection of contrast medium, the peak intensity $(\mathrm{dB})$, the time to peak $(\mathrm{s})$, the intensity gain $(\mathrm{dB})$, the intensity at $60 \mathrm{~s}\left(\mathrm{I}_{60}, \mathrm{~dB}\right)$, and the reduction rate at $60 \mathrm{~s}$ (reduction rate [\%] = $\left[1-\mathrm{I}_{60} /\right.$ peak intensity $\left.] \times 100\right)($ Fig. 1$)$.

\section{Statistical Analysis}

All analyses were performed using the statistical software SAS 9.1.3 (SAS Institute Inc., Cary, NC, USA). The Steel-Dwass test was applied to compare the TIC parameters among the 4 groups. When the $p$ value was $<0.05$, the difference was considered statistically significant. For the diagnosis of pancreatic carcinoma, receiveroperating characteristic (ROC) analysis was performed for all TIC parameters to determine the sensitivity, specificity, and odds ratio using the optimal cutoff value.

\section{Results}

During the study period, 76 consecutive patients with suspect pancreatic masses were enrolled. Of the enrolled patients, 44 were men and 32 women; their mean age was $68.3 \pm 10.2$ years. Table 1 shows the final diagnoses in the 76 patients. There were 41 patients with pancreatic carcinoma, which was diagnosed histologically after surgical resection in 13 cases and EUS-FNA in 28. Six patients with pancreatic neuroendocrine tumor were diagnosed by histological analysis of resected specimens. In the remaining 8 patients, neuroendocrine tumor was diag-
56

Oncology 2017;93(suppl 1):55-60 DOI: $10.1159 / 000481231$
Omoto et al. 
Fig. 1. Quantitative perfusion analysis of contrast-enhanced harmonic endoscopic ultrasonography of a pancreatic carcinoma. A region of interest (yellow circle) is placed over the pancreatic mass. Baseline intensity $(\mathrm{dB})$, echo intensity before injection of contrast agent; peak intensity $(\mathrm{dB})$, echo intensity at the peak; time to peak (s), time from injection of contrast medium to peak intensity; intensity gain, echo intensity gain from baseline to peak intensity; $\mathrm{I}_{60}$, echo intensity $60 \mathrm{~s}$ after injection of contrast medium; reduction rate (\%), the rate of reduction intensity from the peak to 60 s: reduction rate $=\left(1-I_{60} /\right.$ peak intensity) $\times 100)$.

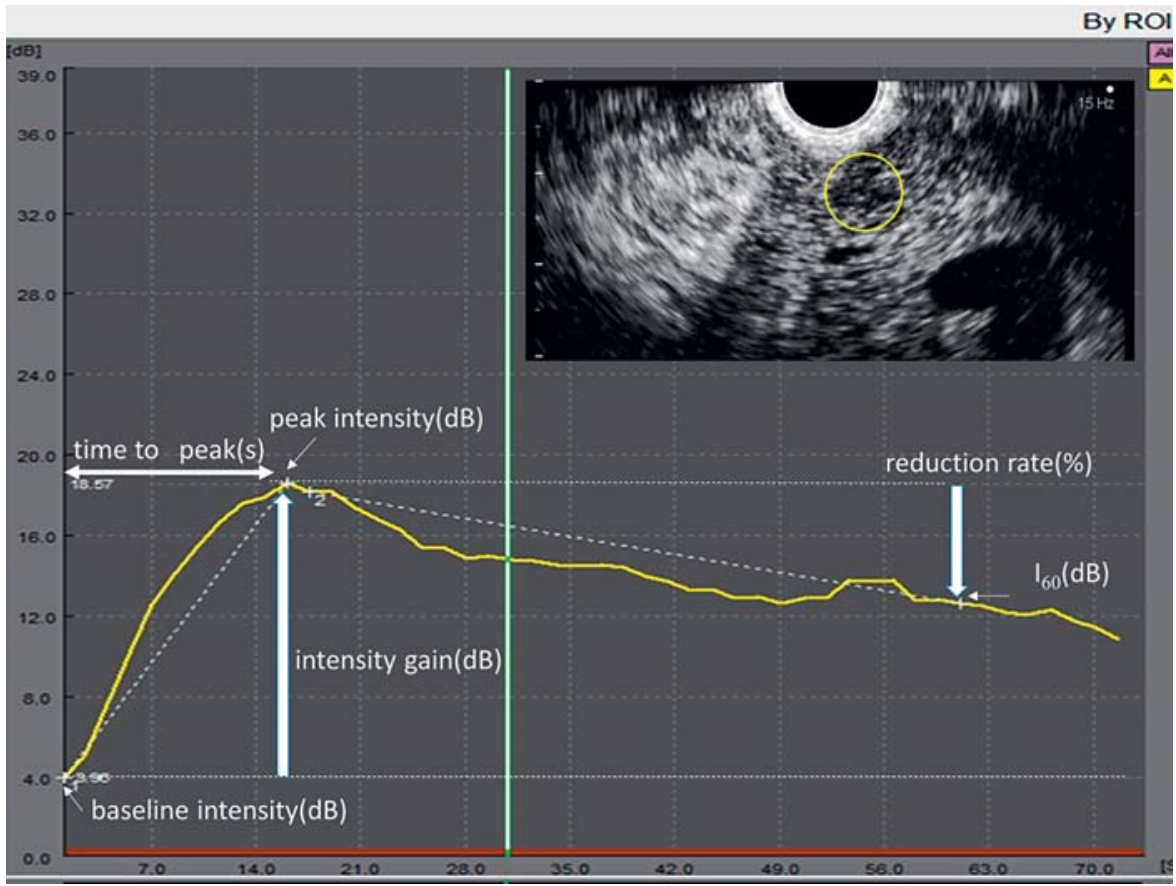

Table 1. Patient characteristics

\begin{tabular}{lc}
\hline Age (mean \pm SD), years & $68.3 \pm 10.2$ \\
Gender (males/females) & $44 / 32$ \\
Maximum tumor diameter (mean \pm SD), mm & $25.9 \pm 16.5$ \\
Final diagnosis, total $n$ ( $n$ surgically resected) & \\
$\quad$ Pancreatic carcinoma & $41(13)$ \\
Inflammatory pseudotumor & $14(0)$ \\
Pancreatic neuroendocrine tumor & $14(6)$ \\
Other tumor & $7(1)$ \\
$\quad$ Pancreatic metastasis from RCC & $4(0)$ \\
Pancreatic metastasis from BC & $2(0)$ \\
Solid pseudopapillary neoplasm & $1(1)$ \\
\hline
\end{tabular}

RCC, renal cell carcinoma; BC, breast carcinoma.

nosed based on EUS-FNA. In all patients with inflammatory pseudotumor, which was diagnosed histologically after EUS-FNA, periodic follow-up with CT and/or EUS revealed a reduction or no change in size. In 10 patients who were suspected of having autoimmune pancreatitis based on the 2011 International Clinical Diagnostic Criteria, tumor reduction was observed after steroid therapy [13]. In the group of 7 patients with other tumors, 4 were pancreatic metastases from renal cell carcinoma, 2 pancreatic metastases from ductal carcinoma of the breast, and 1 was a solid pseudopapillary neoplasm.

Quantitative Analysis of Pancreatic

Tumors
There was no significant difference in baseline intensity among the 4 groups (Fig. 2a). After the injection of contrast medium, values of peak intensity and $\mathrm{I}_{60}$ for the pancreatic carcinoma group were significantly lower than those of the other groups $(p<0.05)$ (Fig. 2b, d). Values of intensity gain for the pancreatic carcinoma group were significantly lower than those of the pancreatic neuroendocrine tumor group and the other tumor groups $(p<$ $0.05)$, although there was no significant difference in intensity gain between pancreatic carcinoma and inflammatory pseudotumor groups (Fig. 2c). Instead, time to peak was significantly longer in the pancreatic carcinoma group than in the other groups $(p<0.05)$ (Fig. 2e). Finally, the reduction rate for pancreatic carcinomas was significantly higher than for pancreatic neuroendocrine tumors $(p<0.01)$ (Fig. 2f).

In ROC analysis for the diagnosis of pancreatic carcinomas (Fig. 3), the areas under the curve for subjective analysis, baseline intensity, peak intensity, intensity gain, $\mathrm{I}_{60}$, time to peak, and reduction rate were $0.817,0.664$, $0.810,0.751,0.845,0.777$, and 0.725 , respectively. According to the ROC data, the optimal cutoff values for these parameters without subjective analysis were 3.2, $15.1,13.7$, and $12.4 \mathrm{~dB}, 12.0 \mathrm{~s}$, and $31.4 \%$, respectively. These cutoffs correspond to the values of sensitivity, specificity, and odds ratio (Table 2). In addition, subjective analyses for the diagnosis of pancreatic carcinomas are

Oncology 2017;93(suppl 1):55-60 57 


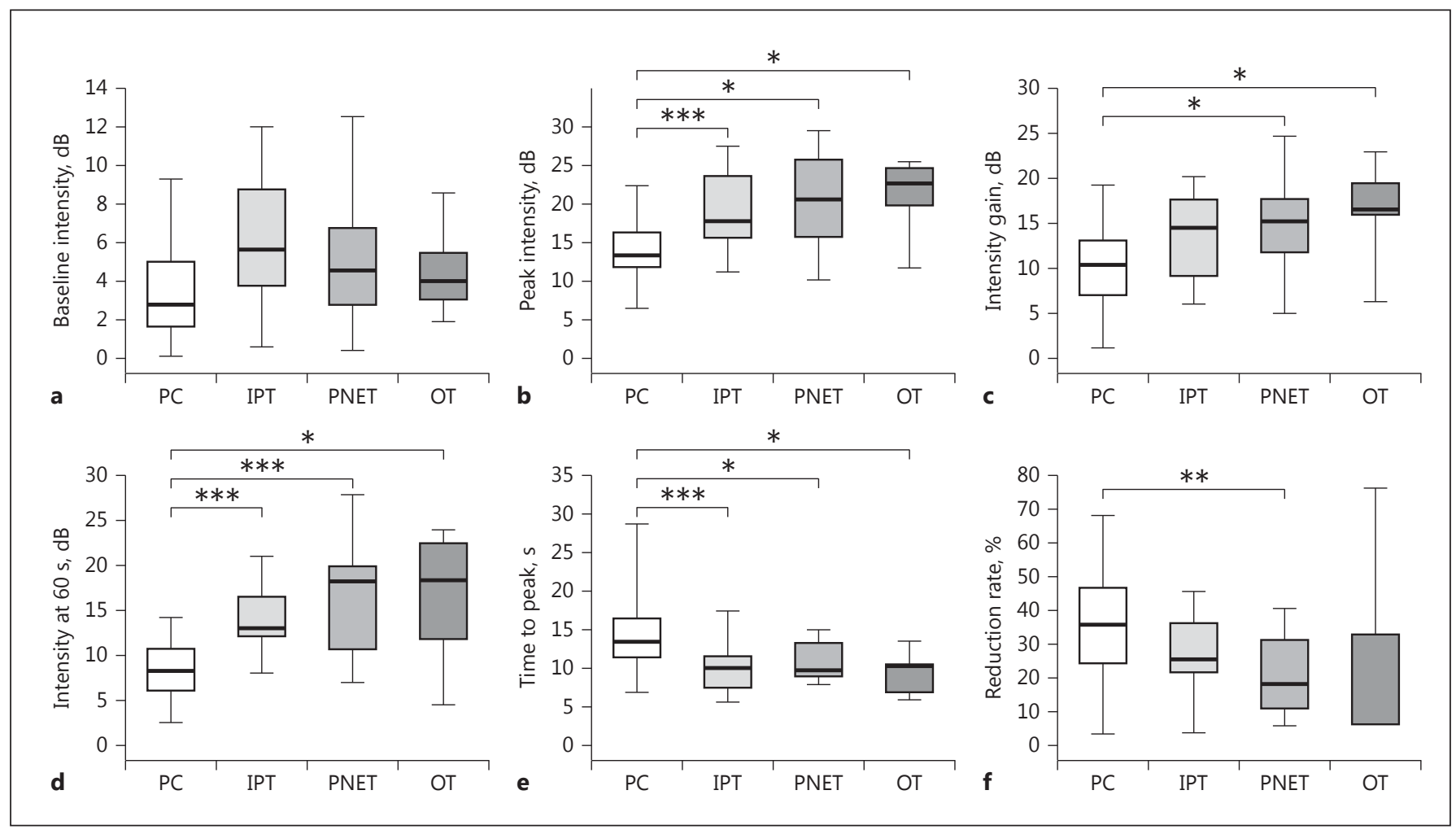

Fig. 2. Comparison of time-intensity curves (TIC) among 4 types of pancreatic tumors. TIC parameters include baseline intensity (a), peak intensity (b), intensity gain (c), intensity gain at $60 \mathrm{~s}(\mathbf{d})$, time to peak (e), and reduction rate $(\mathbf{f})$. PC, pancreatic carcinomas $(n=41)$; IPT, inflammatory pseudotumors $(n=14)$; PNET, pancreatic neuroendocrine tumors $(n=14)$; OT, other tumors $(n=7)$. Values shown are medians (horizontal lines), 25 th and 75 th percentiles (boxes), and ranges (vertical lines). ${ }^{*} p<0.05,{ }^{* *} p<0.01,{ }^{* * *} p<0.005$ (Steel-Dwass test).

Table 2. Receiver-operating characteristic analysis of quantitative perfusion data from contrast-enhanced harmonic endoscopic ultrasonography for the diagnosis of pancreatic carcinoma

\begin{tabular}{lllllr}
\hline Parameter & AUC & Cutoff & Sensitivity & Specificity & Odds ratio \\
\hline Subjective analysis & 0.817 & - & $80.4 \%$ & $82.9 \%$ & 19.938 \\
Baseline intensity & 0.664 & $3.2 \mathrm{~dB}$ & $56.1 \%$ & $74.3 \%$ & 3.691 \\
Peak intensity & 0.810 & $15.1 \mathrm{~dB}$ & $70.7 \%$ & $82.9 \%$ & 11.681 \\
Intensity gain & 0.751 & $13.7 \mathrm{~dB}$ & $82.5 \%$ & $65.7 \%$ & 9.036 \\
I $_{60}$ & 0.845 & $12.4 \mathrm{~dB}$ & $92.7 \%$ & $68.6 \%$ & 27.636 \\
Time to peak & 0.777 & $12.0 \mathrm{~s}$ & $73.2 \%$ & $74.3 \%$ & 7.879 \\
Reduction rate & 0.725 & $31.4 \%$ & $65.9 \%$ & $71.4 \%$ & 4.821 \\
\hline
\end{tabular}

$\mathrm{I}_{60}$, intensity gain at $60 \mathrm{~s}$; AUC, area under the curve.

shown in Table 2 . The sensitivity and specificity of subjective analyses were 80.4 and $82.9 \%$. The $\mathrm{I}_{60}$ (cutoff $=12.4$ $\mathrm{dB}$ ) was the most accurate parameter by ROC analysis for the diagnosis of pancreatic carcinomas with $92.7 \%$ sensitivity, $68.6 \%$ specificity, and an odds ratio of 27.636 .

\section{Discussion}

In this study, various TIC parameters were assessed in pancreatic masses and compared in terms of their ability to diagnose pancreatic carcinoma. In pancreatic carci- 


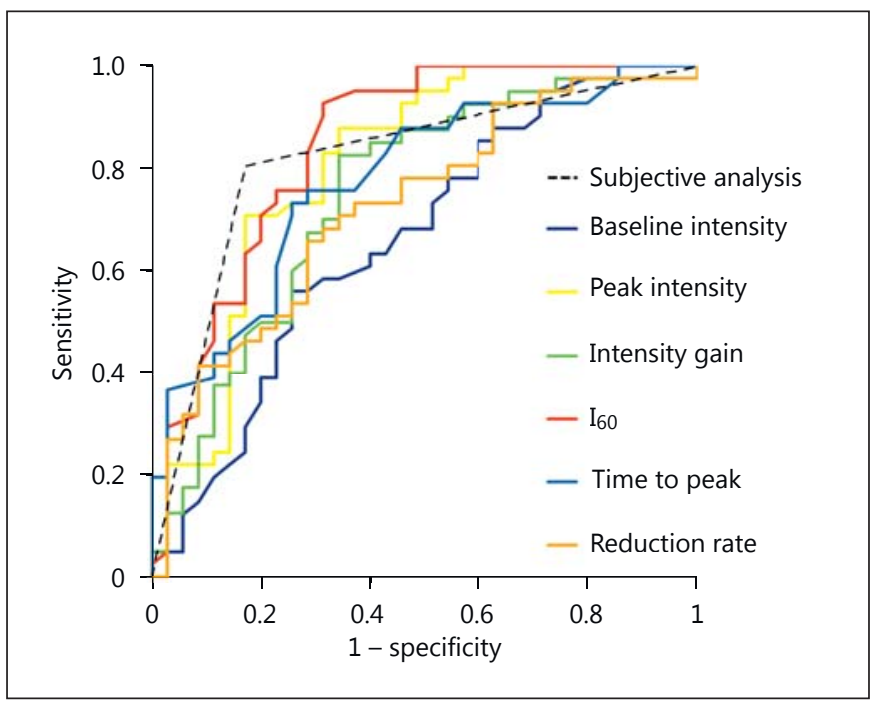

Fig. 3. Receiver-operating characteristic curves for the diagnostic ability of time-intensity curves of contrast-enhanced harmonic endoscopic ultrasonography for pancreatic carcinoma. The areas under the curve for subjective analysis, baseline intensity, peak intensity, intensity gain, intensity gain at $60 \mathrm{~s}\left(\mathrm{I}_{60}\right)$, time to peak, and reduction rate were $0.817,0.664,0.810,0.751,0.845,0.777$, and 0.725 , respectively.

noma patients, TIC patterns markedly differed from the other tumor types. This clinical observation suggests quantitative analysis with $\mathrm{CH}$-EUS is useful in the differential diagnosis of pancreatic masses. There have been some reports on the usefulness of a quantitative analysis of CH-EUS data using TIC in the diagnosis of pancreatic tumors. Seicean et al. [10] compared pancreatic carcinomas and mass-forming chronic pancreatitis in terms of contrast medium uptake and concluded that the uptake ratio (tumor/surrounding tissue) was significantly lower in adenocarcinomas than in pancreatitis, although they analyzed data from 30 patients only. Gheonea et al. [11] assessed various parameters including area under the ROC curve, time to peak, and maximum and median intensity; in their report, the sensitivity and specificity were remarkably high (93.75 and 89.47\%), although it was unclear which parameter was used for diagnostic analysis, and the cutoff values for the parameters were not reported. Imazu et al. [8] found that autoimmune pancreatitis $(n=8)$ and pancreatic carcinomas $(n=18)$ had markedly different TIC. Intensity gain of pancreatic mass lesions in patients with autoimmune pancreatitis was significantly higher than in patients with pancreatic carcinomas (accuracy, 100\%). Săftoiu et al. [12] reported the usefulness of TIC and an automated computer-aided

Quantitative Analysis of Pancreatic Tumors diagnostic system based on their TIC data. Matsubara et al. [9] compared the reduction rate from the peak at 1 min between pancreatic carcinomas $(n=48)$, inflammatory pseudotumors $(n=27)$, and pancreatic neuroendocrine tumors $(n=16)$. In their report, the reduction rate was greatest in pancreatic carcinomas followed by mass-forming pancreatitis, autoimmune pancreatitis, and pancreatic neuroendocrine tumors $(p<0.05)$. However, it is difficult to determine the diagnostic accuracy with quantitative analysis because these previous reports enrolled patients with different kinds of diseases and employed different kinds of parameters. Therefore, our study included all solid tumors and assessed various parameters. There are several parameters for diagnosing pancreatic tumors. From these previous reports [7-12], we chose baseline intensity, peak intensity, intensity gain, $\mathrm{I}_{60}$, time to peak, and reduction rate as parameters for TIC analysis. Peak intensity, intensity gain, and $\mathrm{I}_{60}$ were significantly lower in the pancreatic carcinoma group than in the other groups, while time to peak and reduction rate were higher. These results suggest that pancreatic carcinomas have a slower blood inflow velocity and faster blood outflow velocity, and may reflect the fact that most of these carcinomas have a rich fibrous stroma that usually accounts for the observed hypovascularity [14-16]. However, there are no reports on which TIC parameter represents vessel volume or fibrosis. Further $\mathrm{CH}$-EUS studies on tumors that are all surgically resected are needed to clarify the histological meaning of the 6 TIC parameters used in the present study.

The current study first compared the diagnostic utility of different kinds of TIC parameters using ROC analysis and determined the most reliable TIC parameter. ROC analysis revealed that the best parameter for diagnosing pancreatic carcinomas was $I_{60}$. When the cutoff for $I_{60}$ was set at $12.4 \mathrm{~dB}$, sensitivity and specificity were 92.7 and $68.6 \%$, respectively. $\mathrm{I}_{60}$ had a higher sensitivity than subjective analysis. Eight out of 38 (21\%) correctly diagnosed as pancreatic carcinoma by $\mathrm{I}_{60}$ presented an iso-enhancement pattern by subjective analysis. Despite the evaluation by experts, subjective analysis can be difficult to distinguish from iso- or hypo-enhancement patterns. Therefore, the case of iso-enhancement pattern might be a good adaptation of TIC analysis.

Although $\mathrm{I}_{60}$ was lower than subjective analysis in specificity, $\mathrm{I}_{60}$ was higher than subjective analysis in ROC/ area under the ROC curve. ROC analysis can change the cutoff in all parameters. When the patient cannot receive EUS-FNA because of anticoagulation therapy or intervening vessel, higher specificity is required.

Oncology 2017;93(suppl 1):55-60 DOI: $10.1159 / 000481231$ 
In the present study, a single ROI was placed over the pancreatic tumor. Some reports used a second, parenchymal ROI in the surrounding tissue and measured the value in the tumor relative to the surrounding tissue $[10,11]$. However, when the tumor is large, it is not possible to set 2 ROI (the tumor and the surrounding tissue) on a fixed single image. Moreover, setting 2 ROI may lead to selection bias. Therefore, we chose a single ROI and placed it so as to cover the largest possible tumor area, because the intensity in the tumor is heterogeneous.

Our study has several limitations. A fixed single image without sweep scan technique has to be used in order to obtain TIC. If subjective observation is employed, sweep scanning of the whole lesion with the transducer allows comparison of the tumor and the surrounding tissue in relatively large tumors. It was a retrospective study at a single center, and the sample number was relatively small. Also, some tumors were diagnosed based only on samples obtained by EUS-FNA and follow-up with imaging. A multicenter, prospective study in a larger patient cohort diagnosed only on surgical resection specimens is needed to clarify the diagnostic utility of quantitative perfusion analysis with $\mathrm{CH}$-EUS.

In conclusion, pancreatic carcinoma exhibited markedly different TIC patterns from inflammatory pseudotumors, pancreatic neuroendocrine tumors, and other pancreatic tumors. Thus, quantitative perfusion analysis is useful to differentiate pancreatic carcinomas from other pancreatic masses. $\mathrm{I}_{60}$ is the most accurate parameter.

\section{Acknowledgments}

This study was supported by grants from the Japan Society for Promotion of Science (No. 22590764 and 25461035).

\section{Disclosure Statement}

The authors have no conflicts of interest to declare.

\section{References}

1 Fusaroli P, Spada A, Mancino MG, Caletti G: Contrast harmonic echo-endoscopic ultrasound improves accuracy in diagnosis of solid pancreatic masses. Clin Gastroenterol Hepatol 2010;8:629-634.

2 Kitano M, Kudo M, Yamao K, et al: Characterization of small solid tumors in the pancreas: the value of contrast-enhanced harmonic endoscopic ultrasonography. Am J Gastroenterol 2012;107:303-310.

3 Napoleon B, Alvarez-Sanchez MV, Gincul R, et al: Contrast-enhanced harmonic endoscopic ultrasound in solid lesions of the pancreas: results of a pilot study. Endoscopy 2010; 42:564-570.

4 Kitano M, Sakamoto H, Matsui U, et al: A novel perfusion imaging technique of the pancreas: contrast-enhanced harmonic EUS (with video). Gastrointest Endosc 2008;67: 141-150.

5 Gincul R, Palazzo M, Pujol B, et al: Contrastharmonic endoscopic ultrasound for the diagnosis of pancreatic adenocarcinoma: a prospective multicenter trial. Endoscopy 2014; 46:373-379.
6 Fusaroli P, Kypraios D, Mancino MG, et al: Interobserver agreement in contrast harmonic endoscopic ultrasound. J Gastroenterol Hepatol 2012;27:1063-1069.

7 Kersting S, Konopke R, Kersting F, et al: Quantitative perfusion analysis of transabdominal contrast-enhanced ultrasonography of pancreatic masses and carcinomas. Gastroenterology 2009;137:1903-1911.

8 Imazu H, Kanazawa K, Mori N, et al: Novel quantitative perfusion analysis with contrastenhanced harmonic EUS for differentiation of autoimmune pancreatitis from pancreatic carcinoma Scand J Gastroenterol 2012;47: 853-860.

9 Matsubara H, Itoh A, Kawashima H, et al: Dynamic quantitative evaluation of contrast-enhanced endoscopic ultrasonography in the diagnosis of pancreatic diseases. Pancreas 2011;40:1073-1079.

10 Seicean A, Badea R, Stan-Iuga R, Mocan T, Gulei I, Pascu O: Quantitative contrast-enhanced harmonic endoscopic ultrasonography for the discrimination of solid pancreatic masses. Ultraschall Med 2010;31:571-576.

11 Gheonea D, Streba C, Ciurea T, Săftoiu A: Quantitative low mechanical index contrastenhanced endoscopic ultrasound for the differential diagnosis of chronic pseudotumoral pancreatitis and pancreatic cancer. BMC Gastroenterol 2013;13:2.
12 Săftoiu A, Vilmann P, Dietrich CF, et al: Quantitative contrast-enhanced harmonic EUS in differential diagnosis of focal pancreatic masses (with videos). Gastrointest Endosc 2015;82:59-69.

13 Shimosegawa T, Chari ST, Frulloni L, et al: International consensus diagnostic criteria for autoimmune pancreatitis: guidelines of the International Association of Pancreatology. Pancreas 2011;40:352-358.

14 Numata K, Ozawa Y, Kobayashi N, et al: Contrast-enhanced sonography of pancreatic carcinoma: correlations with pathological findings. J Gastroenterol 2005;40:631-640.

15 Hata H, Mori H, Matsumoto S, et al: Fibrous stroma and vascularity of pancreatic carcinoma: correlation with enhancement patterns on CT. Abdom Imaging 2010;35:172-180.

16 Spivak-Kroizman TR, Hostetter G, Posner R, et al: Hypoxia triggers hedgehog-mediated tumor-stromal interactions in pancreatic cancer. Cancer Res 2013;73:3235-3247. 